\title{
Identification and Detection of Missing RFID Tags using RUN Protocol
}

\author{
Ankita Jade, Nikita Bhirud, Gauri Patwari, Ankita Vaste \\ RMD Sinhgad School of Engg., Computer Department, 111/1,off Mumbai-Bangalore Highway,Warje,Pune-58 \\ ankita.v8996@gmail.com
}

\begin{abstract}
:
As human race or human society is growing, the wildlife animals or wild animals are in danger. But as per natures rule, every living creature on this earth is important and has important role ecosystem. The domestic animals get misplaced sometimes and finding them is a tedious task. RFID and sensors have been deployed to detect and identify missing animals by affixing them with cheap passive RFID tag and monitoring them with RFID readers. So the proposed system will help us to detect and find the missing animals using RUN protocol. RUN protocol uses slotted aloha for communication between tags and readers. It execute multiple frame for different seeds to reduce the effect of unexpected tags and also it reduces the time of missing tag detection and identification. To obtain optimal frame sizes and minimum no of times aloha frames should be executed to mitigate the effect of unexpected tags. RUN protocol works with multiple readers with overlapping region. It identifies $100 \%$ missing tags in the presence of unexpected tags where as other protocol only identifies $60 \%$ of missing tags.
\end{abstract}

Keywords: RFID Monitoring Protocol with Unexpected Tags(RUN), RFID, Missing tag detection, Missing tag identification

\section{Introduction}

RFID is generally used in various application such as Airport, Warehouse, Medical application, shopping malls, inventory control and access control because the cost of trading RFID tags is negligible compared to the value of the product to which they are attached. One of the interesting applications is to detect missing items in large storage. Consider the application airport, using RFID tags it allowing passengers to book seats, print boarding passes and check luggage all in one stop. The printer for the luggage tags equipped with RFID tags for enhanced tracking in minimized headache due to misplace bags for both passengers and airline. In airline RFID also used for medical equipment and personnel, track maintenance, janitorial employees and badges for airport employees. Tags are microchip with an antenna it has limited computation power and communication range. There are two types of tags: (1) Active tags have the own power supply and communication range is much longer than passive tags. (2) Passive tag does not contain any battery, it takes power from reader. When the radio frequency energy from reader encountered by passive RFID, tags forms magnetic field through coiled antenna. There are two types of protocol used for detection and identification of missing tags. (1) Probabilistic protocol, are much faster but only report the missing tags without pinpointing them.

(2) Deterministic protocol, its slower than probabilistic protocol but it points towards missing tags and also return their IDs. Both the protocols have their advantages as well as disadvantages. IN fact, both the protocols are interdependent to each other they should be used together. Probabilistic protocol such as TRP [8][12] and Deterministic protocol such as P-MTI [9] ,SFMTI [5] and IIP there are two limitation of existing protocol it require perfect environments and return id of unexpected tags whose ids are unknown.

In this paper, we are using RUN protocol for identification and detection of missing tags. RUN protocol uses a frame slotted aloha protocol 
specified in the $\mathrm{C} 1 \mathrm{G} 2$ standard. It is very important for RFID protocol to compliant with C1G2 standard

because cheap commercially available off-the shelf (cots) RFID tags follow the C1G2 standard and those protocols does not compliant with $\mathrm{C} 1 \mathrm{G} 2$ standard they require home brewed tags which will cost more and it has a limited settings. AS per the C1G2 standard tags do not transmit their ID until reader specifically asked them to do so. This preserves the privacy in setting. In aloha protocol reader tells the tag a frame size $f$ and a random seed number $\mathrm{R}$. Each tag within a range uses $\mathrm{f}, \mathrm{R}$ and its ID to select slot in frame by calculating hash function h (f, R, ID). Each tag has a counter value with slot number to replay. After each slot the reader first transmits the signals and then each counter value is decremented by one. If all the tags whose counter value is equals to 1 it responds with a pseudo-random number called as RN16. If one or more tags replay in a slot it is called as non empty slot otherwise it is empty slot. location is sent to the user via Email. When the user is offline, the SMS Gateway is used to sent the Text Messages.

Retrieving,Storing,Maintaining, and Accessing the information is all done through RUN protocol.

\section{Dia. System Architecture}

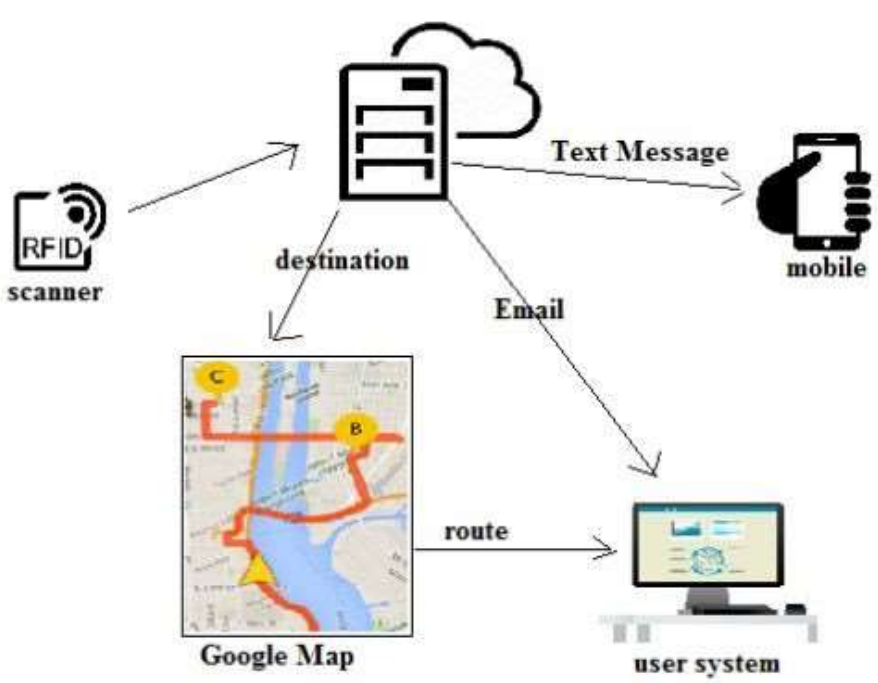

Title, Authors, Body Paragraphs, Sections

Headings and References

Dia. System Architecture
RUN can detect and identify the missing tags. RUN have two functionalities: (1) Used for detection of missing tags calle RUND. RUND executes multiple aloha frames with different seeds. It already knows the ID of all the tags. It identifies which tags will select which slot in each frame. RUND compares the response in each slot of that frame with corresponding slot in pre-computed frames which is used to identify empty and non empty slot, according to that it calculates the optimal value of the system parameter. It is used to minimize the effect of unexpected false positive as well as detection time. (2) Used for identification of missing tags called RUN1. It is used to identify exactly which tag are missing from population of RFID tags, instead of stopping on encountering the first slot that was non empty in pre- computed frames RUN1 continues and executes frames with different seeds on each encounter with an empty slot in frame that was a non empty in pre-computed frame .It marks all the tags that should have responded in that slot as absent. Hence this RUN protocol we are using for tracking domestic animals. In our Proposed work, the tag is attached to the pet animal and the related information about the tag is stored in the database. When the information about the particular tag is to be found then this information is fetched from the database based on tag ID. This information is sent to the user through online and offline line Messages. For online messages SMS Gateway and Google API are used. For offline messages only SMS Gateway is used. Whenever the user request for the missing tag location, the

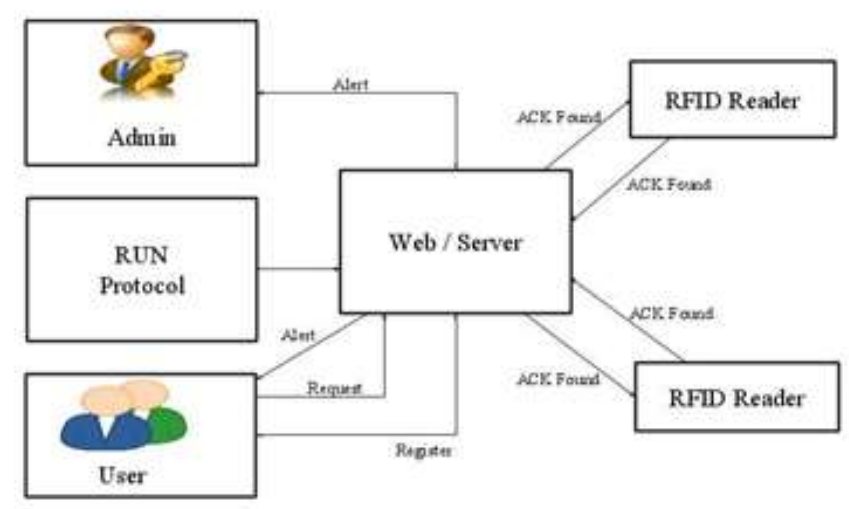

Title and authors

Muhammad Shahzad and Alex X. Liu .In this 
paper, RFID system have been widely developed for various application such as supply chain management, inventory control and access control. The new scheme is invented for estimating tag population size called as average RUN based tag estimation (ART).This technique is based on average run length of ones in the bit string received using slotted aloha. This schema is significantly faster than prior schemes.

Rui Zhang, Yunzhong Liu, Yanchao Zhang, Jinyuan Sun. In this paper they can introduce three novel methods are used for quickly identify the missing tags in large scale RFID system. Slotted ALOHA, anti-collision and MAC protocol can reduce the time for identifying all missing tag up to $75 \%$ with comparison to the previous result.

Lei Yang, Jiaanong Cao, Weiping Zhu and Shaoji Tang. In this paper the RFID tracking which refers to continuously locating a mobile object by attaching it with a RFID reader that communicates with passive RFID tags developed in the environment.one difficult is that the reading are noisy frame in the environment. The particle filter (PF) approach is used for tracking with noisy RFID reading. For this problem the new hybrid method is used which combines particle filter (PF) with Weighted Centroid Localization (WCL) to achieve accuracy with computational cost.

Wai-Kit Sze, Yulin Deng, Wing Cheong Lau, Murali Kodialam, Thyaga Nandagopal and Onching Yue. In this paper scalable, low latency and accurate RFID counting algorithm have been proposed to support more complex query operation.These algorithms do not require explicit identification of individual tags. Due to this it eliminate bottleneck.Proposed design required simple modification of standard RFID tags and readers. It enables fast RFID counting schemes of tag set under non deterministic and unreliable channel.

Xiulong Liu, Keqiu Li, Geyoung Min, Yanming Shen, Alex X.Liu and Wenyu Qu. In this paper, a SFMTI (slot filter based missing tag identification) protocol is proposed which makes expected collision slots in single slot and filters the empty slot. This archive improves time efficiency. A cost effective extension method has been proposed which allows multiple readers to work in parallel mode. Extensive simulation experiments have been conducted which shows that the proposed SFMIT protocol reduces $45 \%$ of the required execution time. The execution time of SFMIT is within a factor of 1.18 .

Tao Li, Shigang Chen and Yibei Ling. In this paper studies the important problem of monitoring a large set if active RFID tags and identifies the missing RFID tags. The protocol reduces the time for detecting the missing tags. A numbers of techniques are introduced in the protocol including hybrid of frame and polling phases, tag removal, collision sensitive tag removal. This technique reduces the time for detection.

Murali Kodialam, Thyaga Nandagopal, Wing Cheong Lau. In this paper, they have proposed a privacy preserving scheme that enables anonymous estimation of another enables anonymous estimation of the cardinality of a dynamic set of RFID tags. The main idea is to avoid explicit identification of tags. The proposed scheme is advantageous because it accurately estimates tag. This scheme performs well changing event, where tag set keep changing.

Chiu C. Tan, Bo Sheng and Qun Li. In this paper, Two protocols are designed for trusted reader (TRP) and untrusted reader (UTRP) for an untrusted reader. A monitoring technique is proposed, which does not require the reader to collect IDs from each RFID tag.

Yuanqing Zheng, Mo Li. In this paper RFID system supports variety of pervasive application. It can be use to label items and enables items level labelling. There are many problem in item level labelling so we used physical layer information which improves the monitoring efficiency.

Zongheng Zhou, Himanshu Gupta, Samir R. Das and Xianjin Zhu. In this paper a centralized algorithm in a slotted time model is developed to read the tag. There are two scenarios (1) where the tag distribution in physical space is unknown. (2) Where the ag distribution is known. The algorithm assumes a planned deployment of readers where it is possible to determine interference and interrogation region of the readers.

Bogdan Carbunar, Murali Krishna Ramanthan, Mehmet Koyuturk, Christop Hoffmann and Ananth Grama. In this paper, They focuses the problem of 
extending the life if the reader network by detecting and temporally turning off redundant reader. To overcome such problem they provides problematic analysis of algorithm.

Wen Lou, Shigang Chen, Yan Qiao, Tao Li. In this paper, the focus is given on reducing the execution time of detection protocol to prevent excessively long protocol execution from interfacing normal inventory operation and they also focus on noise reduction of channels for reliable detection and identification of tags. They propose a protocol that considers both that consider both energy efficiency and time efficiency. They reveal a fundamental energy time trade-off in missing tag detection which can be flexiblycontrol through system parameter to achieve performance. They extend protocols to consider channel error under two different models. It uses multiple hash seeds to provide multiple degree of freedom for the RFID reader to assign tags to singleton slots during which the tags announce their presence in the process of missing tag detection.

\subsection{Overview}

Several protocols [6][8][12][9][5], missing tag detection and identification protocols have been proposed. The common and major drawback of all these protocol is that none of them handle unexpected tags they only handles known tags in the set. The first probabilistic protocol called TRP [8][12] . TRP calculate slots in a frame and compare them with executed slots for detection of missing tags. TRP does not consider false positive from unexpected tags because they assume that the reader already know all IDs in set. This problem is making challenging for RUN protocol because it handles large population (including unexpected tags). All deterministic protocol (IIP, SFMTI [5] , P-MTI [9] ) does not compliant C1G2 standard but the probabilistic protocol TRP is only one compliant with $\mathrm{C} 1 \mathrm{G} 2$ standard with frame size is below 32768 bit. None of the existing probabilistic protocol have been designed to work in multiple reader environment.

Deterministic protocol IIP is non-compliant with C1G2 standard because (1) it required tags to interpret pre-vector frames and replay to reader queries in those frames. (2) it required frame size greater than 32768 bit for large population. (3) it required manufacturers to insert ring of random bits in tag memory at the time of manufacturing. IIP does not handle multiple readers. Deterministic protocol called MTI that handles multiple readers but it not compliant with $\mathrm{C} 1 \mathrm{G} 2$ standard. It first collect all the ids then check which ID is missing but it does not provide accuracy .A Deterministic protocol called SFMTI handles multiple readers but it also not compliant with $\mathrm{C} 1 \mathrm{G} 2$ because it requires stack to interpret non-standardized vectors transmitted by readers before and after selecting slot in a frame. The Deterministic protocol called P-MTI, it use only when there is no unexpected tags because P-MTI needs IDs all tags in the set to calculate sequence of bits for each tag. If the unexpected tags with unknown IDs are present then P-MTI does not able to identify missing tags. The communication channel between readers and tags is reliable that is tags correctly receives queries from readers and the readers correctly detects transmission of RN16 sequence in a slot if one or more tags in the sets transmit in that slot. If channel is unreliable then it use RUN protocol.

\section{Conclusion}

In this paper, we are addressing a problem of detecting and identification of unexpected tags with unknown IDs by using RUN protocol. RUN works with multiple readers with overlapping region and it is easy to deploy. The existing protocol achieves $67 \%$ reliability whereas RUN achieves $100 \%$ reliability in the presence of unexpected tags.

\section{References}

[1] Muhammad Shahzad and Alex X. Liu,"Fast and Accurate Estimation of RFID Tags" IEEE/ACM Transactions on Networking Year: 2015.

[2] R. Zhang, Y. Liu, Y. Zhang, and J. Sun, Fast identification of the missing tags in a large RFID system, in Proc. IEEE SECON, Jun. 2011, pp. 278286.

[3] Lei Yang, Jiaanong Cao, Weiping Zhu and ShaojiTang,"Accurate and Efficient Object Tracking Based on Passive RFID", IEEE Transactions on Mobile Computing, Year:2015.

[4] Wai-Kit Sze, Yulin Deng, Wing Cheong Lau, Murali Kodialam, Thyaga Nandagopal and Onching Yue "Channel Oblivious Counting Algorithms for Large Scale RFID Systems" IEEE Transactions on Parallel and Distributed Systems Year: 2015.

[5] X. Liu, K. Li, G. Min, Y. Shen, A. X. Liu, and W. Qu, "Completely pinpointing the missing 
RFID tags in a time efficient way", IEEE Trans. Computer., vol. 64, no. 1, pp. 8796, Jan. 2015.

[6] Tao Li, Shigang Chen and Yibei Ling \&quot;Efficient Protocols for Identifying the Missing Tags in a Large RFID System",IEEE/ACM Transactions on Networking Year: 2013.

[7] M. Kodialam, T. Nandagopal, and W. C. Lau, "Anonymous tracking using RFID tags," in Proc. 26th IEEE INFOCOM, May 2007, pp. 1217-1225.

[8] C. C. Tan, B. Sheng, and Q. Li, How to monitor for missing RFID tags, in Proc. 28th IEEE ICDCS, Jun. 2008, pp. 295302.

[9] Y. Zheng and M. Li, P-MTI: Physical-layer missing tag identification via compressive sensing, in Proc. IEEE INFOCOM, Apr. 2013, pp. 917925.

[10] Z. Zhou, H. Gupta, S. R. Das, and X. Zhu, "Slotted scheduled tag access in multi-reader RFID systems," in Proc. IEEE ICNP, Oct. 2007, pp. 61-70.

[11] B. Carbunar, M. K. Ramanathan, M. Koyuturk, C.Hoffmann, and A. Grama, "Redundant reader elimination in RFID systems," in Proc. IEEE SECON,Sep. 2005, pp. 176-184

[12] W. Luo, S. Chen, T. Li, and Y. Qiao, "Probabilistic missing-tag detection and energy-time tradeoff in large-scale RFID systems," in Proc.13th ACM Int. Symp. MobiHoc, 2012, pp. 95-104.

[13] Muhammad Shahzad and Alex X. liu, "Fast and Reliable Detection and identification Of Missing RFID tags in the Wild" IEEE transaction on networking, 2016

\section{Author Profile}

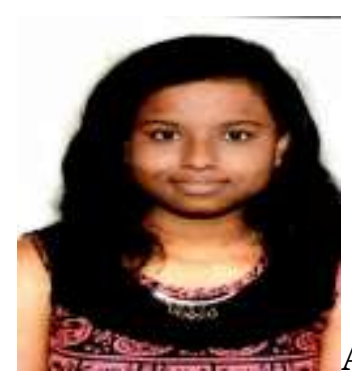

Ankita Jade studied at RMD Sinhgad of Engg., in Computer Engineering from 2015 .

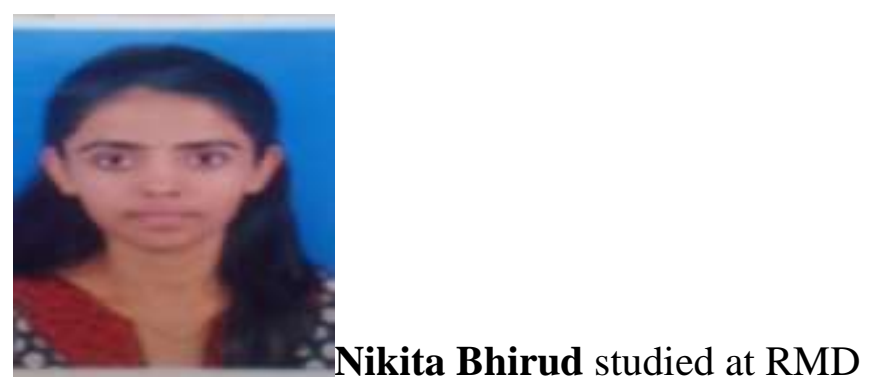

Sinhgad of Engg., in Computer Engineering from 2015 .

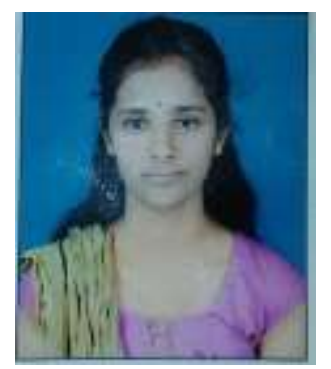

Gauri Patwari studied at RMD Sinhgad of Engg., in Computer Engineering from 2015 .

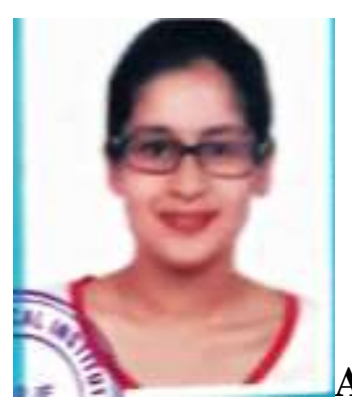

Ankita Vaste studied at RMD Sinhgad of Engg., in Computer Engineering from 2015 